\title{
Perspektiven der universitären Lehrerausbildung im Fach Mathematik für die Sekundarstufen ${ }^{1}$
}

\author{
von Rainer Danckwerts, Susanne Prediger und Eva Vasarhelyi
}

In der Denkschrift zur Lehrerbildung vom Februar 2001 haben sich DMV und GDM gemeinschaftlich für Reformen der universitären Lehrerausbildung ausgesprochen und dazu Vorschläge gemacht. Gerade der fachmathematische Teil der Ausbildung bildet dabei ein Handlungsfeld, in dem die Kooperation von Fachmathematikern und Didaktikern dringend notwendig ist, weil man sich zunehmend bewusst wird, wie stark die eigenen Lernerfahrungen im Studium auch die Vorstellungen vom schulischem Mathematiklernen prägen. Um die gemeinsame Arbeit an diesem Thema anzustoßen, sollen die Probleme und Desiderate hier pointiert formuliert werden.

\section{Das Problem}

Durch den klassischen, systematischen, axiomatischdeduktiven Aufbau der Fachveranstaltungen wird den Studierenden die Wissenschaft Mathematik in der Regel als fertiges, in sich geschlossenes System vermittelt. Dabei spielen die ursprünglichen Problemstellungen, die Prozesse der Begriffsbildung und Theorieentwicklung der jeweiligen Gebiete, nur eine untergeordnete Rolle. Die Methoden der Vermittlung sind einseitig fixiert auf die reine Instruktion durch die klassische Vorlesung.

Die so akzentuierte, traditionelle Fachausbildung ist eher produkt- und weniger prozessorientiert, und sie setzt eher auf die Instruktion durch die Lehrenden als auf die aktive Konstruktion des Wissens durch die Lernenden. In der Balance von Produkt und Prozess sowie von Instruktion und Konstruktion liegt der Schlüssel für eine Verbesserung der fachbezogenen Lehrerausbildung.

\section{Desiderate}

\section{Inhaltlich}

1. Zur prozessorientierten Auffassung der Mathematik als wissenschaftliche Disziplin kann die historisch-genetische Sicht in besonderem Maße beitragen. Daher muss die Geschichte der Mathematik (ideengeschichtlich orientiert und curricular integriert) ihren festen Platz in den Fachstudien haben.

2. $\mathrm{Zu}$ einem gültigen prozessorientierten Bild von Mathematik gehört zwingend die Wechselwirkung zwischen der deduktiv organisierten Mathematik und ihren außermathematischen Anwendungen. Die Anwendungen im Sinne modellbildender Aktivitäten müssen die inhaltliche Auseinandersetzung mit den kanonischen Wissensbeständen der Mathematik durchdringen.

3. Ein fachlich souveräner Umgang mit den Themen des Mathematikunterrichts bahnt sich nicht von selbst an. Hierzu bedarf es eigener elementarmathe-matischer Lehrveranstaltungen, die die Schulmathematik vom höheren (aber nicht primär strukturmathematischen) Standpunkt behandeln und sich insbesondere der Analyse ihres Sinns und ihrer Bedeutung widmen.

\section{Methodisch}

1. Guter Mathematikunterricht bedarf der fruchtbaren Balance zwischen Instruktion (der Schüler durch den Lehrer) und Konstruktion (durch den Schüler selbst). Angehende Mathematiklehrerinnen und -lehrer müssen diese Balance selbst erfahren; sie müssen in ihrem eigenen Lernprozess erleben, wie mathematische Wissensbildung geschieht. Daher gilt es, insbesondere die klassischen Übungen zu den Vorlesungen zu restrukturieren: Sie müssen der identitätsstiftende Ort für die Thematisierung von fachlichen Lernprozessen sein.

2. Für eine aktive Konstruktion des mathematischen Wissens spielen die heuristischen Fähigkeiten eine zentrale Rolle. Geeignete Lehr- und Lernveranstaltungen, etwa Modellierungs- oder ProblemlöseSeminare, müssen fester Bestandteil der Ausbildung sein. Hier können die Prinzipien des aktiventdeckenden Lernens fachbezogen erlebt werden.

\section{Zusammenfassung}

Es geht um einen Paradigmenwechsel im Umgang mit der Mathematik: Nicht nur die Disziplin Mathematik, sondern gleichgewichtig die Beziehung MenschMathematik stehen im Mittelpunkt des Interesses. Dies bedeutet keinesfalls eine Aufweichung wissenschaftlicher Ansprüche an die mathematische Ausbildung, im Gegenteil. Dabei muss die Verbindung zwischen Fach- und Berufsfeldbezug deutlicher werden. Nur ein didaktisch sensibler Umgang mit disziplinärer Mathematik ist anschlussfähig für die Fachdidaktik im engeren Sinne. Das Ziel der Ausbildung liegt im Aufbau eines kognitiven und motivationalen Fundaments, das dem berechtigten Anspruch von Lehramtsstudierenden nach fachbezogener Professionalität Rechnung trägt.

1 Überarbeitete Fassung der in den GDM-Mitteilungen Nr. 76 (Juli 2003) 72 ff erschienenen Version 
Die hier skizzierten notwendigen Veränderungen der fachmathematischen Ausbildung werden in der Implementation viele Herausforderungen mit sich bringen. Ihre Bewältigung bedarf einer engen konzeptionellen Zusammenarbeit der Fachmathematiker und Didaktiker, an den einzelnen Standorten und deutschlandweit. Die dabei auftauchenden Auseinandersetzungen sollten als Chance begriffen werden, gegenseitig voneinander zu lernen.

\section{Ein Beispiel}

Die neue Lehrerprüfungsordnung (LPO) in NRW eröffnet Chancen zur Weiterentwicklung der Lehrerbildung im Sinne des Positionspapiers der GDM. Charakteristische Merkmale der neuen Lehrerausbildungsordnung in NRW sind:

- Neuzuschnitt der Lehrämter mit den Studiengängen GHR (Klasse 1-10) und GYM (Klasse 5-13);

- Stärkere Verzahnung fachwissenschaftlicher, fachdidaktischer und unterrichtspraktischer Studien;

- Modularisierung mit studienbegleitenden Leistungspunkten.

Gerade im Studiengang für das gymnasiale Lehramt (GYM) kann die Verbindung zwischen Fach- und Berufsfeldbezug entschiedener als bisher verwirklicht werden, da auf die Klassenstufen 5-13 (und nicht nur 11-13) vorbereitet werden soll.

Dazu gehört neben dem Kern des klassischen Kanons die Konzeption von elementarmathematischen (nicht fachdidaktischen) Lehrveranstaltungen, die inhaltlich auf die Schulmathematik in den beiden Sekundarstufen gerichtet sind. Solche Module stehen unter dem Motto „Mittel- und Oberstufenmathematik vertieft verstehen“. Sie werden ergänzt durch kohärente fachdidaktische und mathematikgeschichtliche Veranstaltungen.

Die in der nebenstehenden Abbildung wiedergegebene Übersicht zeigt eine modulare Struktur mit diesem Anspruch (realisiert an der Universität Siegen).

\section{Literatur}

P. Borneleit, R. Danckwerts, H.-W. Henn, H.-G. Weigand: Expertise zum Mathematikunterricht in der gymnasialen Oberstufe. In: JMD 22 (2001), Heft 1, S. 73-90.

G. Stroth, G. Törner, R. Scharlau (DMV), W. Blum, K. Reiss, (GDM): Denkschrift zur Lehrerbildung, Februar 2001.

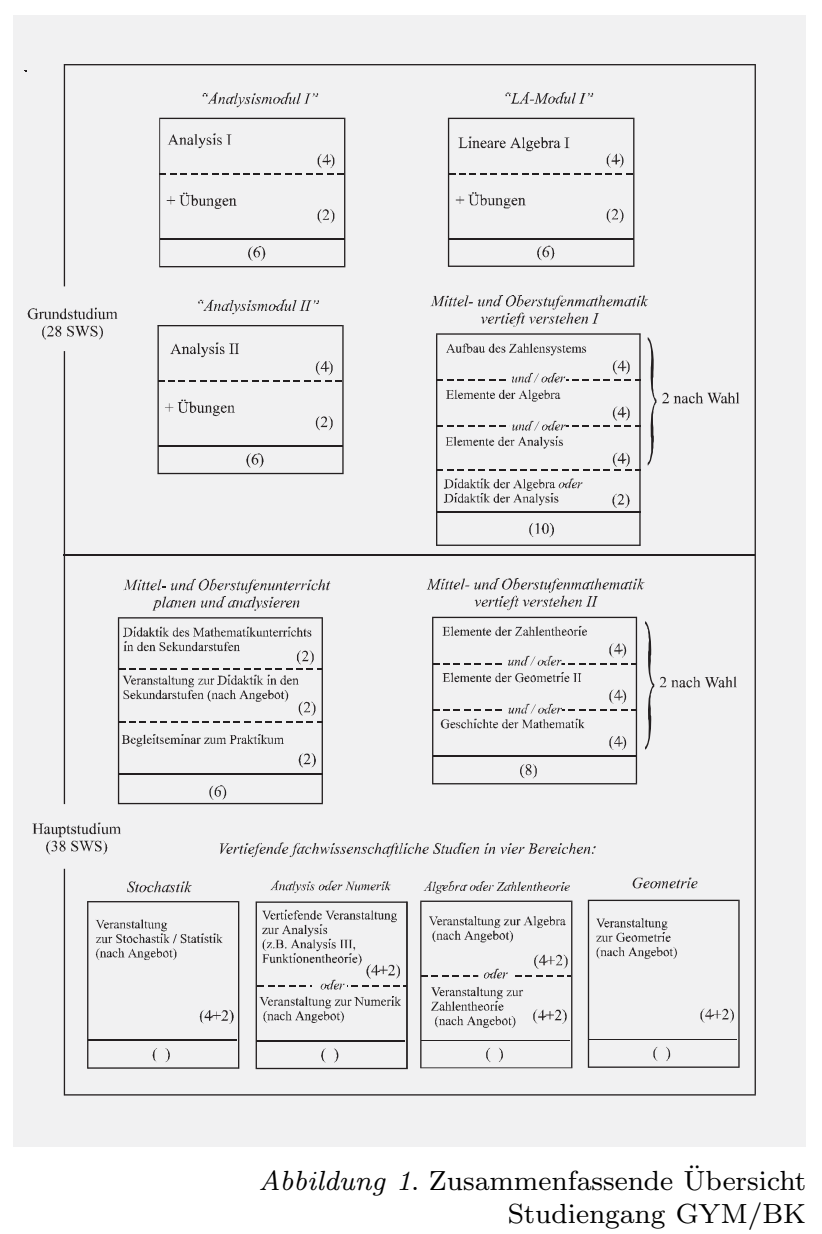

Adressen der Autoren

Prof. Dr. Rainer Danckwerts

Didaktik der Mathematik - Fachbereich 6

Universität-Gesamthochschule Siegen

Walter-Flex-Straße 3

57068 Siegen

danckwerts@mathematik. uni-siegen.de

Prof. Dr. Susanne Prediger

FB Mathematik/Informatik

Universität Bremen

Bibliothekstraße 1

28359 Bremen

prediger@math.uni-bremen.de

Dr. Eva Vasarhelyi

Didaktik der Mathematik

Universität Budapest

vasar@ludens.elte.hu 